\title{
Heavy Eye Syndrome Mimicking Abducens Nerve Palsies
}

\author{
Caberry W. Yu, Jonathan A. Micieli (D)
}

Key words: Heavy eye syndrome, myopia, strabismus, cranial nerve palsy

doi:10.1017/cjn.2020.85

Can J Neurol Sci. 2020; 47: 683-684

A 70-year-old woman was seen in neuro-ophthalmology consultation for a progressive right esotropia. She had a past medical history of asthma and osteoporosis, and her ocular history was significant for pathological myopia (spherical equivalent of $-23.00 \mathrm{D}$ OD and $-21.00 \mathrm{D}$ OS), bilateral cataract surgeries, and laser retinopexy for retinal tears. Twenty-five years prior to presentation, she developed binocular horizontal diplopia and was found to have a limitation of abduction to $60 \%$ and $80 \%$ of normal in the right and left eyes, respectively, by a previous neuro-ophthalmologist. She developed a progressive maculopathy in the right eye secondary to myopia, resulting in the resolution of double vision. The resolution occurred because the double vision was binocular in nature and the loss of vision in her right eye functioned similar to monocular occlusion. However, she noticed that her right eye continued to turn in toward her nose.

On examination, her best-corrected visual acuities were counting fingers OD and 20/30 OS. She had a large right esotropia and hypotropia in primary position. Extraocular motility examination revealed limitation of abduction and elevation, greater in the right eye compared to the left (Figure 1). Magnetic resonance imaging (MRI) of the orbits revealed superotemporal prolapse of the globes, medial displacement of superior rectus (SR) muscles, and inferior displacement of lateral rectus (LR) muscles bilaterally (Figure 2). The imaging features were characteristic of heavy eye syndrome (HES). She elected for observation as she did not experience bothersome symptoms.

HES is a rare but well-recognized cause of adult strabismus and diplopia. ${ }^{1,2}$ It is found in patients with high axial myopia and results from the disruption of the LR-SR band, a ligament that supports the vertical position of the LR muscle. ${ }^{1,3}$ This leads to an inferior shift of the LR pulley and a medial shift of the SR with superotemporal prolapse of the myopic globe. ${ }^{4,5}$ Inferior displacement of the LR pulley results in alteration of its force to depress the eye, while nasal translocation of the SR pulley results in alternation of its force to adduct the eye. ${ }^{1}$ Since there is no strong abducting force, an esotropia results.

HES or highly myopic strabismus should be suspected in any patient with a new esotropia, limitation of abduction, and high myopia. An MRI of the orbits should be performed to confirm the diagnosis and avoid unnecessary investigations for abducens nerve palsy, which may also exist in myopic patients. HES is amenable to surgical correction, which involves joining the posterior bellies of the SR and LR muscles, restoring the normal anatomic positions of the extraocular muscles, and the abducting
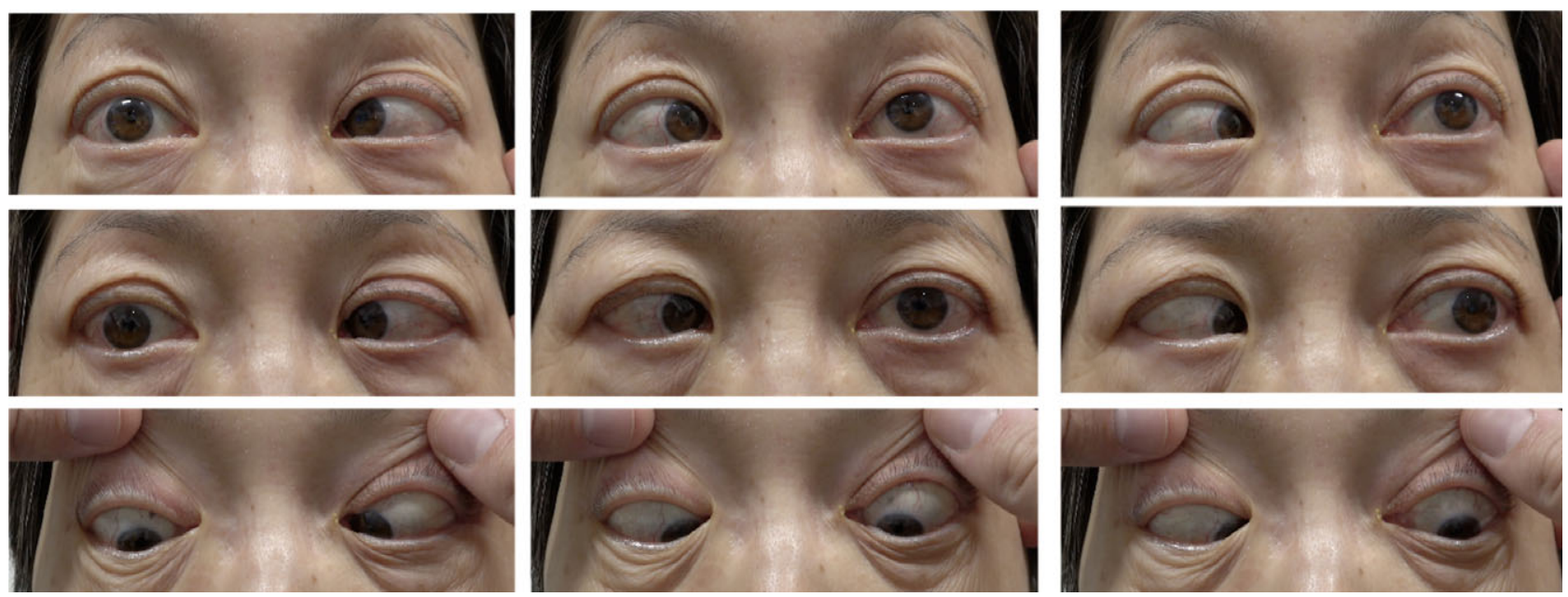

Figure 1: Eye movements in nine cardinal positions of gaze demonstrating a right esotropia and hypotropia in primary position, a limitation of abduction and elevation in both eyes.

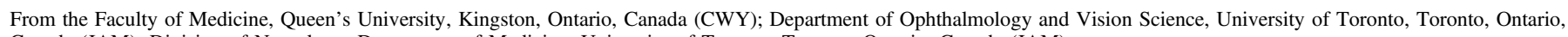
Canada (JAM); Division of Neurology, Department of Medicine, University of Toronto, Toronto, Ontario, Canada (JAM)

Received March 1, 2020. Final Revisions Submitted April 16, 2020. Date of Acceptance April 20, 2020.

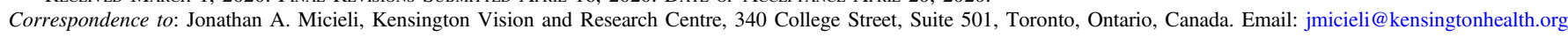




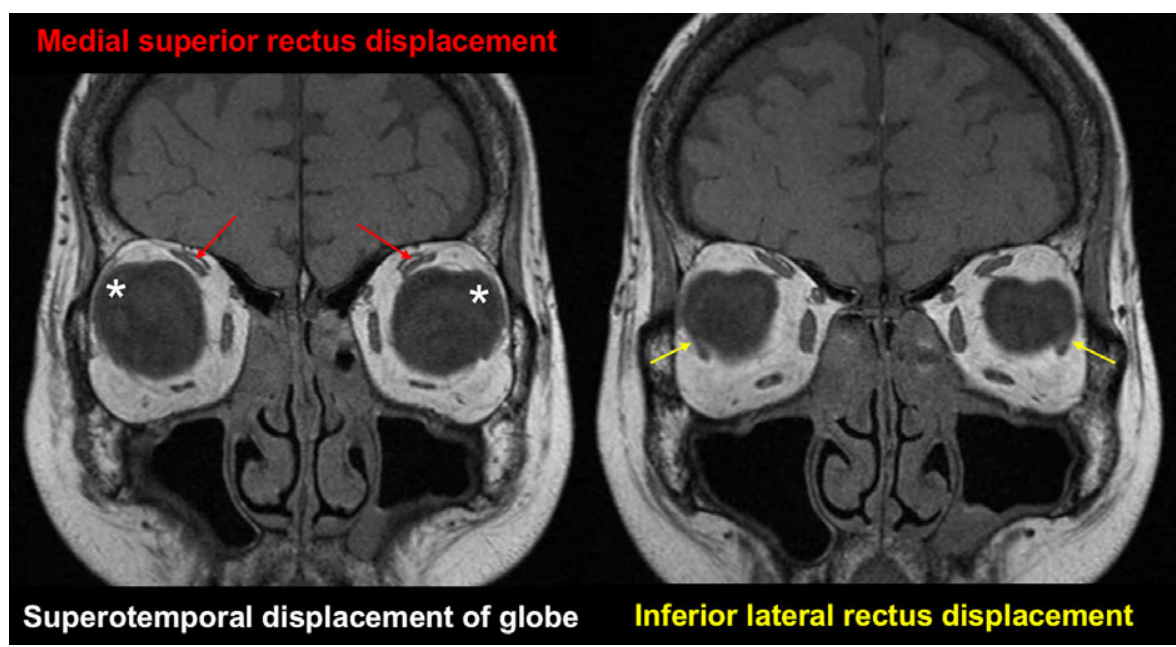

Figure 2: Coronal T1 magnetic resonance imaging of the orbits demonstrating superotemporal displacement of the globes, medial superior rectus displacement, and inferior lateral rectus displacement.

and supraducting forces. ${ }^{6}$ The globe is also able to freely move in the intraconal space, and mechanical disruptions to eye movements are eliminated.

In conclusion, high axial myopia may result in anatomical changes in the orbit, leading to an esotropia and hypotropia. The diagnosis can be confirmed with orbital imaging in the coronal plane to confirm medial displacement of the SR, inferior displacement of the LR, and superior displacement of the globe.

\section{DiscLOSURES}

The authors have no conflicts of interest to declare.

\section{Statement of Authorship}

CWY and JAM were both involved in the project conception, authorship, and editing.

\section{REFERENCES}

1. Peragallo JH, Pineles SL, Demer JL. Recent advances clarifying the etiologies of strabismus. J Neuro-Ophthalmol. 2015;35(2): 185-93. doi: 10.1097/WNO.0000000000000228

2. Narayana KM, Nusbaum A, Galetta SL. Myopia/heavy eye syndrome: a rare cause of horizontal diplopia. Neurology. 2015; 85(8):737-38. doi: 10.1212/WNL.0000000000001872

3. Kono R, Poukens V, Demer JL. Quantitative analysis of the structure of the human extraocular muscle pulley system. Investig Ophthalmol Vis Sci. 2002;43(9):2923-32.

4. Maiolo C, Fresina M, Campos EC. Role of magnetic resonance imaging in heavy eye syndrome. Eye. 2017;31(8):1163-67. doi: 10.1038/eye. 2017.48

5. Demer JL, von Noorden GK. High myopia as an unusual cause of restrictive motility disturbance. Surv Ophthalmol. 1989; 33(4):281-84. doi: 10.1016/0039-6257(82)90154-0

6. Yamaguchi M, Yokoyama T, Shiraki K. Surgical procedure for correcting globe dislocation in highly myopic strabismus. Am J Ophthalmol. 2010;149(2):341-46. doi: 10.1016/j.ajo.2009.08. 035 